\title{
RIESZ TRANSFORMS ON GENERALIZED HEISENBERG GROUPS AND RIESZ TRANSFORMS ASSOCIATED TO THE CCR HEAT FLOW
}

\author{
Françoise Lust-Piquard
}

\begin{abstract}
Let $1<q<\infty$. We prove that the Riesz transforms $R_{k}=X_{k} L^{-\frac{1}{2}}$ on a generalized Heisenberg group $G$ satisfy $\left\|\left(\sum_{k=1}^{K}\left|R_{k}(f)\right|^{2}\right)^{\frac{1}{2}}\right\|_{L^{q}(G)} \leq C(q, J)\|f\|_{L^{q}(G)}$ where $K, J$ are respectively the dimensions of the first and second layer of the Lie algebra of $G$. We prove similar inequalities on Schatten spaces $S^{q}(H)$, with dimension free constants, for Riesz transforms associated to commuting inner $*$-derivations $D_{k}$ and a suitable substitute of the square function. An example is given by the derivations associated to $n$ commuting pairs of operators $\left(P_{j}, Q_{j}\right)$ on a Hilbert space $H$ satisfying the canonical commutation relations $\left[\mathrm{P}_{j}, Q_{j}\right]=i I_{H}$.
\end{abstract}

\section{General introduction}

This paper is divided in two parts: we solve similar problems in two different settings, using similar methods inspired by the first part of [P1], which contains a proof of the following classical inequalities $[\mathbf{S}]$ : for $1<q<\infty$ and $f \in \mathcal{D}\left(\mathbb{R}^{n}\right)$,

$$
D_{q}\|f\|_{L^{q}\left(\mathbb{R}^{n}\right)} \leq\left\|\left(\sum_{k=1}^{n}\left|R_{k}(f)\right|^{2}\right)^{\frac{1}{2}}\right\|_{L^{q}\left(\mathbb{R}^{n}\right)} \leq C_{q}\|f\|_{L^{q}\left(\mathbb{R}^{n}\right)}
$$

where

$$
R_{k}(f)=\frac{\partial}{\partial x_{k}} L^{-\frac{1}{2}}(f), \quad L=-\sum_{k=1}^{n} \frac{\partial^{2}}{\partial x_{k}^{2}}
$$

and the constants do not depend on $n$.

2000 Mathematics Subject Classification. 43A80, 46L50, 46 L57.

Key words. Heat operator, Riesz transforms, H-groups, commuting *-inner derivations. 
The first part deals with Riesz transforms acting on $L^{q}(G)$, where $G$ is a generalized Heisenberg group, and owes a lot to [CMZ].

The second part deals with Riesz transforms acting on the Schatten space $\mathcal{S}_{q}(H)$, associated to commuting $*$-inner derivations on the algebra $K(H)$ of compact operators on a Hilbert space $H$; an example is given by the inner derivations defined by $\left(P_{j}, Q_{j}\right)_{j=1}^{n}$, where $P_{j}, Q_{j}$ satisfy the canonical commutation relation $\left[Q_{j}, P_{j}\right]=i I_{H}$ and the other commutators are zero.

We already used Pisier's method in other settings, see e.g. [LP2], [LP3]. However the difficulties arise at different steps in different applications.

Since the two settings we consider are very different, we present more precise introductions in each part.

Acknowledgement. We thank V. Georgescu for fruitful discussions on the setting of the second part. We also thank the referee for detecting a gap in the proof of Theorem 1.b in an earlier version of the paper.

\section{Riesz transforms on generalized Heisenberg groups}

\subsection{Introduction.}

For any stratified Lie group $G$, we denote by $X_{1}, \ldots, X_{K}$ a basis of the top layer of the Lie algebra $\mathcal{G}$ of $G$, by

$$
L=-\sum_{j=1}^{K} X_{j}^{2}
$$

the subelliptic Kohn Laplacian on $G$, by

$$
R_{k}=X_{k} L^{-\frac{1}{2}}, \quad 1 \leq k \leq K,
$$

the Riesz transforms. The boundedness of each $R_{k}$ on $L^{q}(G), 1<q<\infty$, is known: the classical proof uses the homogeneity of its kernel and the singular integral results of [FS, Chapter 6]; see also Lemma 2 below. Our interest in this paper is to look for dimension free inequalities involving $\left\|\left(\sum_{k=1}^{K}\left|R_{k}(f)\right|^{2}\right)^{\frac{1}{2}}\right\|_{L^{q}(G)}$.

We first consider the case where $G$ is a Heisenberg group $\mathbb{H}_{n}$ and give a simpler proof of the main result of $[\mathbf{C M Z}]$. We extend this result to the generalized Heisenberg groups $\mathbb{H}_{K, J}$ defined by Kaplan $[\mathbf{K}]$; they are particular step two stratified Lie groups, and $K, J$ denote respectively the dimensions of the first and second layer in the Lie algebra of $\mathbb{H}_{K, J}$. The Heisenberg group $\mathbb{H}_{n}$ is the same as $\mathbb{H}_{2 n, 1}$. 
Theorem 1. Let $1<q<\infty, \frac{1}{q}+\frac{1}{q^{\prime}}=1$.

a) $[\mathbf{C M Z}]$ There exist constants $C_{q}$ such that, for every $n \in \mathbb{N}^{*}$ and every $f \in \mathcal{D}\left(\mathbb{H}_{n}\right)$,

$$
C_{q^{\prime}}^{-1}\|f\|_{L^{q}\left(\mathbb{H}_{n}\right)} \leq\left\|\left(\sum_{k=1}^{2 n}\left|R_{k}(f)\right|^{2}\right)^{\frac{1}{2}}\right\|_{L^{q\left(\mathbb{H}_{n}\right)}} \leq C_{q}\|f\|_{L^{q}\left(\mathbb{H}_{n}\right)} .
$$

b) The same holds for generalized Heisenberg groups $\mathbb{H}_{K, J}$, with constants $C(q, J)$ which depend on $q$ and $J$ but not on $K$.

It is a standard fact (see e.g. [CMZ]) that, in the above formula, for any Lie group $G$, the lefthand side inequality for $q$ is an easy consequence of the righthand side one for $q^{\prime}, \frac{1}{q}+\frac{1}{q^{\prime}}=1$.

Theorem 1 relies on Christ's study $[\mathbf{C}]$ of Hilbert transforms along curves for homogeneous nilpotent Lie groups, and on the use of dilations $\delta_{t}$ in order to get an expression of the convolution operator $e^{-\frac{1}{2} t^{2} L}$ involving the (heat) kernel $p$ of $e^{-\frac{1}{2} L}$, namely (see Lemma 2 a))

$$
e^{-\frac{1}{2} t^{2} L}(f)(\gamma)=\int_{G} f\left(\gamma \delta_{t} g^{-1}\right) p(g) d g .
$$

Theorem 1 also uses a formula (Lemma 2 b) below)

$$
\sqrt{2 \pi} X L^{-\frac{1}{2}}(f)(\gamma)=\int_{G} F(\gamma, g)(X p)(g) d g
$$

which holds on every stratified group $G, X$ lying in the first layer of $\mathcal{G}, F$ being a Hilbert transform of $f$. These ingredients are already in $[\mathbf{C M Z}]$; they are reminiscent of the method of $[\mathbf{P 1}]$.

In the proof of Theorem $1 \mathrm{a}$ ), our improvement upon $[\mathbf{C M Z}]$ is that we do not use the explicit formula of $p$ and avoid computations. Denoting $p=p^{(n)}$ when $G=\mathbb{H}_{n}$, we use only the following properties:

(i) $[\mathbf{F S}] p$ is a positive function lying in $\mathcal{S}(G)$, and $\int_{G} p d g=1$,

(ii) $p^{(n)}\left(x_{1}, \ldots, y_{n}, u\right)$ is radial with respect to $\left(x_{1}, \ldots, y_{n}\right)$, i.e. depends on $(r, u)$,

(iii) $p^{(n)}\left(x_{1}, \ldots, y_{n}, u\right)=p^{(1)}\left(x_{1}, y_{1}, u\right) *_{u} \ldots *_{u} p^{(1)}\left(x_{n}, y_{n}, u\right)$, where $*{ }_{u}$ denotes convolution in $\mathbb{R}$ with respect to the variable $u$. Property (ii) is used through the observation that

$$
X_{j} p^{(n)}=x_{j} \frac{1}{r} \frac{\partial p^{(n)}}{\partial r}+2 y_{j} \frac{\partial p^{(n)}}{\partial u} .
$$

In the proof of Theorem $1 \mathrm{~b}$ ) we use the analogue for $\mathbb{H}_{K, J}$. 
We recall that the heat kernels (i.e. the kernels of $e^{-t L}$ ) on $\mathbb{H}_{n}$ and the non isotropic Heisenberg groups, or rather their Fourier transform with respect to $u$, were first computed in $[\mathbf{G}]$ and $[\mathbf{H}]$, then in several subsequent papers, by rather complicated methods; the heat kernels on general step two stratified groups were computed in $[\mathbf{C Y}]$. A more explicit formula for generalized Heisenberg groups $\mathbb{H}_{K, J}$ is given in $[\mathbf{R}]$, using the result for $\mathbb{H}_{n}$. Let us mention our computation of the heat kernels for isotropic or non isotropic Heisenberg groups and the free step two stratified groups $N_{n, 2}[\mathbf{L P 1}]$, which is simpler than the previous ones and relies on the common starting point of $[\mathbf{C M Z}]$ and the present paper, namely formula (1).

\subsection{Notation.}

For a background on stratified groups (which are particular homogeneous groups) we refer to $[\mathbf{F S}$, Chapter I]. We consider stratified Lie groups $G$ equipped with their Haar measure, denoted by $d g$ or $d \gamma$, which is Lebesgue measure on the underlying space $\mathbb{R}^{d} \cdot \mathcal{D}(G)$ denotes the space of $\mathcal{C}^{\infty}$ compactly supported functions on $G, \mathcal{S}(G)$ denotes the Schwartz class. The convolution of two functions $f, h$ lying in $\mathcal{S}(G)$ is defined by

$$
f * h(\gamma)=\int_{G} f\left(\gamma g^{-1}\right) h(g) d g
$$

The Lie algebra of left invariant vector fields on $G$ is denoted by $\mathcal{G}$. For $X \in \mathcal{G}$,

$$
X(f * p)=f * X p .
$$

The first layer of $\mathcal{G}$ is the linear subspace which spans $\mathcal{G}$ as a Lie algebra. We denote by $\sigma$ the automorphism of $G$, corresponding to the automorphism $\sigma$ of $\mathcal{G}$ whose action on the first layer is

$$
\sigma: X \longrightarrow-X \text {. }
$$

$G$ is equipped with a dilation $\delta_{t}, t>0$, corresponding to the automorphism of $\mathcal{G}$ whose action on the first layer is

$$
\delta_{t} X=t X \text {. }
$$

The induced action on functions $f: G \rightarrow \mathbb{R}$ is denoted by $\left(\delta_{t} f\right)(g)=$ $f\left(\delta_{t} g\right)$, and $[\mathbf{F S}, \mathrm{I} \mathbf{C}]$

$$
X \delta_{t} f=t \delta_{t}(X f) .
$$

A stratified Lie group $G$ is said to be step two if the central layer of $\mathcal{G}$ is the second one; denoting by $X_{1}, \ldots, X_{K}$ a basis of the first layer, and by $U_{1}, \ldots, U_{J}$ a basis of the second layer, it means that all 
commutators $\left[X_{j}, X_{k}\right]$ belong to the linear span of the $U_{j}$ 's and the other commutators are zero. Every $g \in G$ is defined in a unique way by $g=\exp \left(\sum_{k=1}^{K} x_{k} X_{k}+\sum_{j=1}^{J} u_{j} U_{j}\right)$ and we denote $g=(x, u)=$ $\left(x_{1}, \ldots, x_{K}, u_{1}, \ldots, u_{J}\right)$. In this setting, the Haar measure on $G$, i.e. the Lebesgue measure on $\mathbb{R}^{K+J}$, is also denoted by $d x d u$. In particular, $\sigma g=\sigma(x, u)=(-x, u)$, hence $\sigma g^{-1}=(x,-u)$, and $\delta_{t}(x, u)=\left(t x, t^{2} u\right)$.

We will use the following easy, but crucial, result which is standard when $J=0$ and $\psi$ is the gaussian density on $\mathbb{R}^{K}$.

Lemma 2. Let $\psi$ be a measurable function: $\mathbb{R}^{K+J} \rightarrow \mathbb{R}$ such that $\int_{\mathbb{R}^{J}}|\psi(x, u)| d u$ only depends on $|x|, x=\left(x_{k}\right)_{k=1}^{K} \in \mathbb{R}^{K}$. Then

a) if $1 \leq q<\infty, a_{k} \in \mathbb{C}$,

$$
\left\|\sum_{k=1}^{K} a_{k} x_{k}\right\|_{L^{q}(|\psi| d x d u)}=\left(\sum_{k=1}^{K}\left|a_{k}\right|^{2}\right)^{\frac{1}{2}}\left\|x_{1}\right\|_{L^{q}(|\psi| d x d u)} ;
$$

b) if $1<q<\infty, \frac{1}{q}+\frac{1}{q^{\prime}}=1$, for every $h \in L^{q}(|\psi| d x d u)$,

$$
\left(\sum_{k=1}^{K}\left|\int_{\mathbb{R}^{K+J}} x_{k} h \psi d x d u\right|^{2}\right)^{\frac{1}{2}} \leq\left\|x_{1}\right\|_{L^{q^{\prime}(|\psi| d x d u)}}\|h\|_{L^{q}(|\psi| d x d u)} .
$$

Proof: For short, we write $L^{q}(|\psi|)$ instead of $L^{q}(|\psi| d x d u)$. We use polar coordinates in $\mathbb{R}^{K}$, namely $a=\left(a_{1}, \ldots, a_{K}\right)=|a| w, x=|x| v=r v$, with $w, v \in \Sigma_{K}, v=\left(v_{1}, \ldots, v_{K}\right)$, here $\Sigma_{K}$ denotes the unit sphere of $\mathbb{R}^{K}$ and $d \sigma_{K}$ is the uniform measure on it, with total mass the area of $\Sigma_{K}$.

a) Since $\int_{\mathbb{R}^{J}}|\psi| d u$ only depends on $r$,

$$
\begin{aligned}
\left\|\sum_{k=1}^{K} a_{k} x_{k}\right\|_{L^{q}(|\psi|)}^{q} & =|a|^{q} \int_{0}^{\infty} r^{q}\left(\int_{\mathbb{R}^{J}}|\psi| d u\right) r^{K-1} d r \int_{\Sigma_{K}}|\langle w, v\rangle|^{q} d \sigma_{K}(v) \\
& =|a|^{q} \int_{0}^{\infty} r^{q}\left(\int_{\mathbb{R}^{J}}|\psi| d u\right) r^{K-1} d r \int_{\Sigma_{K}}\left|v_{1}\right|^{q} d \sigma_{K}(v) \\
& =|a|^{q}\left\|x_{1}\right\|_{L^{q}(|\psi|)}^{q} .
\end{aligned}
$$


b) This follows from a): indeed, by Hölder inequality,

$$
\begin{aligned}
\left(\sum_{k=1}^{K}\left|\int x_{k} h \psi d x d u\right|^{2}\right)^{\frac{1}{2}} & =\sup _{|a|=1}\left|\int\left(\sum_{k=1}^{K} a_{k} x_{k}\right) h \psi d x d u\right| \\
& \leq\|h\|_{L^{q}(|\psi|)} \sup _{|a|=1}\left\|\sum_{k=1}^{K} a_{k} x_{k}\right\| \|_{L^{q^{\prime}}(|\psi|)} \\
& =\|h\|_{L^{q}(|\psi|)}\left\|x_{1}\right\|_{L^{q^{\prime}(|\psi|)}} .
\end{aligned}
$$

Let $G$ be a stratified Lie group; to $g \in G$ we associate the curve: $\mathbb{R} \rightarrow G$

$$
\begin{array}{ll}
g(t)=\delta_{t} g & \text { for } \quad t \geq 0 \\
g(t)=\delta_{|t|} \sigma g & \text { for } \quad t<0 .
\end{array}
$$

In particular, if $G$ is step two, the curve associated to $g=(x, u)$ is

$$
g(t)=\left(t x, t^{2} u\right), \quad t \in \mathbb{R} .
$$

The Hilbert transform of $f$ along the curve $g(t)$ is

$$
\begin{aligned}
F(\gamma, g) & =\mathrm{pv} \int_{-\infty}^{\infty} f\left(\gamma g(t)^{-1}\right) \frac{d t}{t} \\
& =\lim _{\varepsilon \rightarrow 0^{+}} \int_{\varepsilon<|t|<\varepsilon^{-1}} f\left(\gamma g(t)^{-1}\right) \frac{d t}{t} \\
& =\lim _{\varepsilon \rightarrow 0^{+}} F(\gamma, g, \varepsilon) .
\end{aligned}
$$

The function $F(\gamma, g)$ is well defined on $G \times G$ because $f \in \mathcal{D}(G)$. $F(\gamma, g, \varepsilon)$ is called the truncated Hilbert transform.

The map $h_{t}^{g}: f \rightarrow f\left(. g(t)^{-1}\right), t \in \mathbb{R}, L^{\infty}(G) \rightarrow L^{\infty}(G)$, is a $*$-homomorphism of the *-algebra $L^{\infty}(G)$, but $\left\{h_{t}^{g}\right\}_{t \in \mathbb{R}}$ is not a one parameter group. This makes an important difference with the settings of [LP1], [LP2], [LP3] and the second part of this paper.

The next result comes from [CMZ, Proof of Lemmas 1 and 5]. For the sake of completeness we give a more precise proof. 
Lemma 3. Let $G$ be a stratified Lie group and $f \in \mathcal{D}(G)$. Then

$$
e^{-\frac{1}{2} t^{2} L}(f)(\gamma)=\int_{G} f\left(\gamma \delta_{t} g^{-1}\right) p(g) d g, \quad t>0
$$

and, for $X$ in the first layer of $\mathcal{G}, d \gamma$ a.s.,

$$
\sqrt{2 \pi} X L^{-\frac{1}{2}}(f)(\gamma)=\int_{G} F(\gamma, g)(X p)(g) d g
$$

where $F(\gamma, g)$ is the Hilbert transform of $f$ along the curve $g(t), g \in G$.

For $1<q<\infty$, the Riesz transforms satisfy

$$
\left\|\sqrt{2 \pi} X L^{-\frac{1}{2}}(f)\right\|_{L^{q}(G)} \leq h_{q}\|X p\|_{L^{1}(G)}\|f\|_{L^{q}(G)} .
$$

Proof: Formula (1) holds true for $t=1$ by definition of $p$. By (4) (see also $[\mathbf{L P 1}])$,

hence, by (5),

$$
e^{-\frac{1}{2} t^{2} L}=\delta_{t^{-1}} e^{-\frac{1}{2} L} \delta_{t}
$$

(1) $\quad e^{-\frac{1}{2} t^{2} L}(f)(\gamma)=\delta_{t^{-1}}\left[\left(f \circ \delta_{t}\right) * p(\gamma)\right]=\int_{G} f\left(\gamma \delta_{t} g^{-1}\right) p(g) d g$

By (5), (1) and (3),

$$
\left.X e^{-\frac{1}{2} t^{2} L}(f)=t^{-1} \delta_{t^{-1}} X\left[\left(f \circ \delta_{t}\right) * p\right)\right]=t^{-1} \delta_{t^{-1}}\left[\left(f \circ \delta_{t}\right) *(X p)\right] .
$$

The automorphism $\sigma$ maps $L$ to $L$, so $p=p \circ \sigma$ and

$$
X p=X(p \circ \sigma)=-(X p) \circ \sigma .
$$

For $h \in \mathcal{D}(G)$, since $\sigma^{2} g=g$ and $\sigma$ is measure preserving,

$$
\begin{aligned}
h *(X p)(\gamma) & =\int_{G} h\left(\gamma g^{-1}\right)(X p)(g) d g \\
& =-\int_{G} h\left(\gamma g^{-1}\right)(X p)(\sigma g) d g \\
& =-\int_{G} h\left(\gamma \sigma g^{-1}\right)(X p)(g) d g .
\end{aligned}
$$

In particular, for $f \in \mathcal{D}(G)$,

$$
2 X e^{-\frac{1}{2} t^{2} L}(f)(\gamma)=t^{-1} \int_{G}\left[f\left(\gamma \delta_{t} g^{-1}\right)-f\left(\gamma \delta_{t} \sigma g^{-1}\right)\right](X p)(g) d g
$$

Since

$$
\sqrt{\frac{\pi}{2}} X L^{-\frac{1}{2}}(f)=\int_{0}^{\infty} X e^{-\frac{1}{2} t^{2} L}(f) d t
$$


we get

$$
\sqrt{2 \pi} X L^{-\frac{1}{2}}(f)(\gamma)=\int_{0}^{\infty}\left(\int_{G}\left[f\left(\gamma \delta_{t} g^{-1}\right)-f\left(\gamma \delta_{t} \sigma g^{-1}\right)\right](X p)(g) d g\right) \frac{d t}{t} .
$$

Since $p \in \mathcal{S}(G), X p \in L^{1}(G)$, and formula (2) now comes from the subsequent Lemma $4 \mathrm{~b})$.

By (2) and Hölder inequality with $\frac{1}{q}+\frac{1}{q^{\prime}}=1, d \gamma$ a.s.,

$$
\sqrt{2 \pi}\left|X L^{-\frac{1}{2}}(f)(\gamma)\right| \leq\|F(\gamma, .)\|_{L^{q}(|X p| d g)}\|X p\|_{L^{1}(G)}^{\frac{1}{q^{\prime}}} .
$$

By the subsequent Lemma 4 a),

$$
\|F(\gamma, g)\|_{L^{q}\left(d \gamma, L^{q}(|X p| d g)\right)} \leq h_{q}\|f\|_{L^{q}(G)}\|X p\|_{L^{1}(G)}^{\frac{1}{q}},
$$

which ends the proof.

The next lemma comes from $[\mathbf{C}]$; it is already used in this setting in $[\mathbf{C M Z}]$, see [CMZ, Lemma 5].

Lemma 4. Let $G$ be a stratified Lie group and $f \in \mathcal{D}(G)$. For $g \in G$ let $F(\gamma, g)$ be the Hilbert transform of $f$ along the curve $g(t)$ and let $\psi \in L^{1}(G)$. Then

a) for $1<q<\infty$, there exists a constant $h_{q}$ such that

$$
\|F\|_{L^{q}(d \gamma \otimes|\psi| d g)} \leq h_{q}\|f\|_{L^{q}(d \gamma)}\|\psi\|_{1}^{\frac{1}{q}} .
$$

b)

$$
\begin{aligned}
\int_{G} F(\gamma, g) & \psi(g) d g \\
& =\int_{0}^{\infty}\left[\int_{G}\left(f\left(\gamma \delta_{t} g^{-1}\right)-f\left(\gamma \delta_{t} \sigma g^{-1}\right)\right) \psi(g) d g\right] \frac{d t}{t}, \quad d \gamma \text { a.s. }
\end{aligned}
$$

Proof: a) When $g$ runs through $G$, the corresponding family of curves $g(t)$ satisfies the assumptions of [C, pp. 579 and 594]. By the main result of $[\mathbf{C}]$, if $1<q<\infty$, there exists a constant $h_{q}$ such that, for every $g \in G$,

$$
\|F(., g)\|_{L^{q}(G)} \leq h_{q}\|f\|_{L^{q}(G)} .
$$

This proves a) by integration with respect to $g$ and Fubini theorem.

b) For every $g \in G$, the truncated Hilbert transform $F(\gamma, g, \varepsilon)$ along the curve $g(t)$ satisfies [C, Lemma 6.3]

$$
\|F(., g)-F(., g, \varepsilon)\|_{L^{2}(G)} \longrightarrow_{\varepsilon \rightarrow 0^{+}} 0
$$


and

$$
\sup _{\varepsilon>0}\|F(., g, \varepsilon)\|_{L^{2}(G)} \leq h_{2}\|f\|_{L^{2}(G)} .
$$

Hence, since $\psi \in L^{1}(G)$, the dominated convergence theorem implies

$$
\begin{aligned}
& \lim _{\varepsilon \rightarrow 0^{+}}\left\|\int_{G}(F(\gamma, g)-F(\gamma, g, \varepsilon)) \psi(g) d g\right\|_{L^{2}(G)} \\
\leq & \lim _{\varepsilon \rightarrow 0^{+}} \int_{G}\|F(., g)-F(., g, \varepsilon)\|_{L^{2}(G)}|\psi(g)| d g=0 .
\end{aligned}
$$

This implies b), because, by Fubini theorem, $d \gamma$ a.s.,

$$
\begin{aligned}
\int_{G} F(\gamma, g, \varepsilon) \psi(g) d g \\
=\int_{\varepsilon<t<\varepsilon^{-1}}\left[\int_{G}\left(f\left(\gamma \delta_{t} g^{-1}\right)-f\left(\gamma \delta_{t} \sigma g^{-1}\right)\right) \psi(g) d g\right] \frac{d t}{t}
\end{aligned}
$$

Proof of Theorem 1: We treat Heisenberg groups first because the proof in this case is simpler and the idea is more apparent.

a) When $G=\mathbb{H}_{n}$, we denote $p=p^{(n)}$. The group law on $\mathbb{H}_{n}$ is

$$
\begin{aligned}
\gamma g & =\left(x_{1}, y_{1}, \ldots, x_{n}, y_{n}, u\right)\left(x_{1}^{\prime}, y_{1}^{\prime}, \ldots, x_{n}^{\prime}, y_{n}^{\prime}, u^{\prime}\right) \\
& =\left(x_{1}+x_{1}^{\prime}, y_{1}+y_{1}^{\prime}, \ldots, x_{n}+x_{n}^{\prime}, y_{n}+y_{n}^{\prime}, u+u^{\prime}+2 \sum_{j=1}^{n}\left(y_{j} x_{j}^{\prime}-x_{j} y_{j}^{\prime}\right)\right) .
\end{aligned}
$$

By definition, for $g=\left(x_{1}, \ldots, y_{n}, u\right)$,

$$
\begin{aligned}
\left(X_{j} p^{(n)}\right)(g) & =\frac{\partial p^{(n)}}{\partial x_{j}}(g)+2 y_{j} \frac{\partial p^{(n)}}{\partial u}(g) \\
\left(Y_{j} p^{(n)}\right)(g) & =\frac{\partial p^{(n)}}{\partial y_{j}}(g)-2 x_{j} \frac{\partial p^{(n)}}{\partial u}(g) .
\end{aligned}
$$

The Laplacian is given by

$$
-L=\sum_{j=1}^{n}\left[\frac{\partial^{2}}{\partial x_{j}^{2}}+\frac{\partial^{2}}{\partial y_{j}^{2}}+4\left(x_{j}^{2}+y_{j}^{2}\right) \frac{\partial^{2}}{\partial u^{2}}+4\left(x_{j} \frac{\partial^{2}}{\partial y_{j} \partial u}-y_{j} \frac{\partial^{2}}{\partial x_{j} \partial u}\right)\right]
$$

hence commutes with rotations on $\left(x_{1}, \ldots, y_{n}\right)$. It follows that $p^{(n)}$ is radial with respect to $\left(x_{1}, \ldots, y_{n}\right)$, hence so are $\frac{\partial p^{(n)}}{\partial u}$ and the function

$$
\frac{1}{r} \frac{\partial p^{(n)}}{\partial r}=\frac{1}{x_{j}} \frac{\partial p^{(n)}}{\partial x_{j}}=\frac{1}{y_{j}} \frac{\partial p^{(n)}}{\partial y_{j}}
$$


where $r=\left(\sum_{j=1}^{n} x_{j}^{2}+y_{j}^{2}\right)^{\frac{1}{2}}$. Rewriting

$$
\left(X_{j} p^{(n)}\right)(g)=x_{j} \frac{1}{r} \frac{\partial p^{(n)}}{\partial r}(g)+2 y_{j} \frac{\partial p^{(n)}}{\partial u}(g)
$$

we have, by (2), $d \gamma$ a.s.,

$\sqrt{2 \pi} X_{j} L^{-\frac{1}{2}}(f)(\gamma)=\int_{G} x_{j} F(\gamma, g) \frac{1}{r} \frac{\partial p^{(n)}}{\partial r}(g) d g+2 \int_{G} y_{j} F(\gamma, g) \frac{\partial p^{(n)}}{\partial u}(g) d g$ and a similar formula for $Y_{j} L^{-\frac{1}{2}}(f)$. Denoting for $1 \leq j \leq n$

$$
\begin{aligned}
& R_{j}=X_{j} L^{-\frac{1}{2}} \\
& R_{n+j}=Y_{j} L^{-\frac{1}{2}}
\end{aligned}
$$

by triangular inequality in $l_{2 n}^{2}$ and Lemma 2 applied on $\mathbb{R}^{2 n+1}, d \gamma$ a.s.,

$$
\text { (6) } \begin{aligned}
\sqrt{2 \pi}\left(\sum_{k=1}^{2 n}\left|R_{k}(f)(\gamma)\right|^{2}\right)^{\frac{1}{2}} & \leq\|F(\gamma, .)\|_{L^{q}\left(\frac{1}{r}\left|\frac{\partial p(n)}{\partial r}\right| d g\right)}\left\|x_{1}\right\|_{L^{q^{\prime}}\left(\frac{1}{r}\left|\frac{\partial p(n)}{\partial r}\right| d g\right)} \\
& +2\|F(\gamma, .)\|_{L^{q}\left(\left|\frac{\partial p(n)}{\partial u}\right| d g\right)}\left\|x_{1}\right\|_{L^{q^{\prime}\left(\left|\frac{\partial p(n)}{\partial u}\right| d g\right)}} .
\end{aligned}
$$

Since $L$ is the sum of $-\left(X_{j}^{2}+Y_{j}^{2}\right), 1 \leq j \leq n$, which act on different set of variables except for the central $u[\mathbf{C M Z}$, p. 371], (see also the proof of $\mathrm{b}$ ) below)

$$
p^{(n)}\left(x_{1}, \ldots, y_{n}, u\right)=p^{(1)}\left(x_{1}, y_{1}, u\right) *_{u} \ldots *_{u} p^{(1)}\left(x_{n}, y_{n}, u\right) .
$$

Since $p^{(n)} \geq 0$ for every $n$,

$$
\frac{1}{r}\left|\frac{\partial p^{(n)}}{\partial r}\right|=\left|\frac{1}{x_{1}} \frac{\partial p^{(n)}}{\partial x_{1}}\right| \leq\left|\frac{1}{x_{1}} \frac{\partial p^{(1)}}{\partial x_{1}}\right| *_{u} p^{(n-1)} .
$$

Since $\int_{\mathbb{H}_{n-1}} p^{(n-1)}(g) d g=1$, for $q \geq 0$,

$$
\int_{\mathbb{H}_{n}}\left|x_{1}\right|^{q}\left|\frac{1}{r} \frac{\partial p^{(n)}}{\partial r}\right| d g \leq \int_{\mathbb{R}^{3}}\left|x_{1}\right|^{q}\left|\frac{1}{x_{1}} \frac{\partial p^{(1)}}{\partial x_{1}}\right| d x_{1} d y_{1} d u=A_{q} .
$$

Since $p^{(1)}$ belongs to $\mathcal{S}\left(\mathbb{R}^{3}\right), A_{q}$ is obviously finite for $q \geq 1$, and also for $q=0$. Indeed

$$
\left\|\frac{1}{x_{1}} \frac{\partial p^{(1)}}{\partial x_{1}}\right\|_{L^{1}\left(\mathbb{R}^{3}\right)}=2 \pi \int_{\mathbb{R}^{+} \times \mathbb{R}}\left|\frac{\partial p^{(1)}}{\partial r}\right| d r d u
$$


and $\left|\frac{\partial p^{(1)}}{\partial r}\right|=\left(\left|\frac{\partial p^{(1)}}{\partial x_{1}}\right|^{2}+\left|\frac{\partial p^{(1)}}{\partial y_{1}}\right|^{2}\right)^{\frac{1}{2}}$. Similarly

$$
\int_{\mathbb{H}_{n}}\left|x_{1}\right|^{q}\left|\frac{\partial p^{(n)}}{\partial u}\right| d g \leq \int_{\mathbb{R}^{3}}\left|x_{1}\right|^{q}\left|\frac{\partial p^{(1)}}{\partial u}\right| d x_{1} d y_{1} d u=B_{q}
$$

and $B_{q}$ is finite for $q \geq 0$.

Integrating (6) with respect to $\gamma$, we get by Lemma 4 a)

$$
\begin{aligned}
\sqrt{2 \pi} & \left\|\left(\sum_{k=1}^{2 n}\left|R_{k}(f)\right|^{2}\right)^{\frac{1}{2}}\right\|_{L^{q}\left(\mathbb{H}_{n}\right)} \leq A_{q^{\prime}}^{\frac{1}{q^{\prime}}}\|F\|_{L^{q}\left(d \gamma \otimes\left|\frac{1}{r} \frac{\partial p^{(n)}}{\partial r}\right| d g\right)} \\
& +2 B_{q^{\prime}}^{\frac{1}{q^{\prime}}}\|F\|_{L^{q}\left(d \gamma \otimes\left|\frac{\partial p(n)}{\partial u}\right| d g\right)} \leq h_{q}\|f\|_{L^{q}\left(\mathbb{H}_{n}\right)}\left(A_{q^{\prime}}^{\frac{1}{q^{\prime}}} A_{0}^{\frac{1}{q}}+2 B_{q^{\prime}}^{\frac{1}{q^{\prime}}} B_{0}^{\frac{1}{q}}\right)
\end{aligned}
$$

which gives the righthand side inequality in the statement of the theorem. Denoting

$$
\mathcal{R}: f \longrightarrow\left(R_{k}(f)\right)_{k=1}^{K} \text {, }
$$

this means that $\mathcal{R}$ is bounded: $L^{q}(G) \rightarrow L^{q}\left(G, l_{2 n}^{2}\right)$, and so is

$$
\mathcal{R}^{*}:\left(h_{k}\right)_{k=1}^{2 n} \longrightarrow \sum_{k=1}^{2 n} R_{k}^{*}\left(h_{k}\right), \quad L^{q^{\prime}}\left(G, l_{2 n}^{2}\right) \longrightarrow L^{q^{\prime}}(G) .
$$

Since $f=\sum_{k=1}^{2 n} R_{k}^{*} R_{k}(f)=\mathcal{R}^{*} \mathcal{R}(f)$ and $\left\|\mathcal{R}^{*}\right\|_{q \rightarrow q}=\|\mathcal{R}\|_{q^{\prime} \rightarrow q^{\prime}}$, we get in the standard way the lefthand side inequality:

$$
\|f\|_{L^{q}(G)} \leq\left\|\mathcal{R}^{*}\right\|_{q \rightarrow q}\left\|\left(\sum_{k=1}^{2 n}\left|R_{k}(f)\right|^{2}\right)^{\frac{1}{2}}\right\|_{L^{q}(G)} .
$$

b) When $G=\mathbb{H}_{K, J}$, we recall $[\mathbf{K}],[\mathbf{C D K R}]$ that its Lie algebra $\mathcal{G}$ is equipped with a scalar product, so that the first layer $\mathcal{V}$ is a real vector space with dimension $K$, orthogonal to the second layer $\mathcal{Z}$ with dimension $J$. We denote by $|X|$ the norm on $\mathcal{G}$ defined by the scalar product.

The specific property is that, for every $X$ with norm 1 in $\mathcal{V}$, the operator $\operatorname{ad}_{X}$ defined on $\mathcal{V}$ by

$$
\operatorname{ad}_{X}: Y \longrightarrow[X, Y]
$$

is an isometry from $E=\left(\operatorname{kerad}_{X}\right)^{\perp} \subset \mathcal{V}$ onto $\mathcal{Z}$. In particular, $E$ has dimension $J$ and $\left|\operatorname{ad}_{X}(Y)\right|=\left|P_{E}(Y)\right|$ where $P_{E}$ is the orthogonal projection onto $E$. 
Let us now precise the structure of $\mathcal{V}+\mathcal{Z}$ both as a Hilbert space and a Lie algebra. For $U \in \mathcal{Z}$ let $\Phi_{U}: \mathcal{V} \rightarrow \mathcal{V}$ be the linear operator defined by

$$
\left\langle\Phi_{U}(X), X^{\prime}\right\rangle=\left\langle U,\left[X, X^{\prime}\right]\right\rangle .
$$

In particular, $\left(\Phi_{U}\right)^{*}=-\Phi_{U}$, so that, if $F \subset \mathcal{V}$ is an invariant subspace for $\Phi_{U}, F^{\perp}$ is also invariant. The operator $\Phi: \mathcal{Z} \rightarrow B(\mathcal{V})$ defined by $U \rightarrow$ $\Phi_{U}$ satisfies

$$
\Phi_{U} \Phi_{U^{\prime}}+\Phi_{U^{\prime}} \Phi_{U}=-2\left\langle U, U^{\prime}\right\rangle \operatorname{Id} \mathcal{V} .
$$

In particular, if $U_{1}, \ldots, U_{J}$ is an orthonormal basis of $\mathcal{Z},\left(\Phi_{U j}\right)_{j=1}^{J}$ are unitary skew-adjoint anticommuting operators on $\mathcal{V}$. In more sophisticated words (see [BTV, $3.1 .2]$ ), denoting by $q$ the quadratic form defined on $\mathcal{Z}$ by $q(U)=-\langle U, U\rangle, \Phi$ induces a representation of the real Clifford algebra $\mathcal{C}_{J}$ built on $(\mathcal{Z}, q)$ into $B(\mathcal{V})$. In $\mathcal{C}_{J}$ one has $U^{2}=-$ Id for every $U \in \mathcal{Z}$ with norm 1 , so that $\mathcal{C}_{J}$ is the linear span of Id and $U_{i_{1}} U_{i_{2}} \ldots U_{i_{n}}$, $1 \leq i_{1}<i_{2}<\cdots<i_{n} \leq J$, and has dimension $2^{J}$. $\Phi$ is a direct sum of irreducible representations of $\mathcal{C}_{J}$. By the classification of the Clifford algebras $\mathcal{C}_{J}$, their irreducible representations are as follows (see e.g. [ $\mathbf{H u}$, Chapter 11] or [ABS, Part I]):

If $J \neq 3(\bmod 4)$, there is only one (up to equivalence) irreducible representation of $\mathcal{C}_{J}$. Hence $\mathcal{V}$ must be splitted as a hilbertian sum $\mathcal{V}=\mathcal{V}_{1} \oplus \cdots \oplus \mathcal{V}_{N}$ where the spaces $\mathcal{V}_{l}$ have the same dimension $K_{J}$ and are invariant under all $\Phi_{U}, U \in \mathcal{Z}$. In particular, the $\mathcal{V}_{l}$ 's are commuting copies with isomorphic Lie structure. We may choose orthonormal basis $\left(X_{(l-1) K_{J}+i}\right)_{i=1}^{K_{J}}$ of the $\mathcal{V}_{l}$ 's such that $\left[X_{(l-1) K_{J}+i}, X_{(l-1) K_{J}+h}\right]$ does not depend on $l$, for $1 \leq i, h \leq K_{J}$.

If $J \equiv 3(\bmod 4)$, there are two non equivalent irreducible representations of $\mathcal{C}_{J}$, with the same dimension $K_{J} . \mathcal{V}$ must be splitted as a hilbertian sum $\mathcal{V}_{1} \oplus \cdots \oplus \mathcal{V}_{k} \oplus \mathcal{V}_{k+1}^{\prime} \oplus \cdots \oplus \mathcal{V}_{N}^{\prime}$ where the $\mathcal{V}_{l}$ 's (resp. the $\mathcal{V}_{l}^{\prime}$ 's) are commuting copies with isomorphic Lie structure, and the $\mathcal{V}_{l}$ 's commute with the the $\mathcal{V}_{l}^{\prime}$ 's. We choose orthonormal basis of the $\mathcal{V}_{l}$ 's (resp. the $\mathcal{V}_{l}^{\prime}$ 's) as above.

Conversely, by $[\mathbf{K}]$, if $\mathcal{Z}, \mathcal{V}$ are real finite dimensional Hilbert spaces, from a linear isometry $\Phi: \mathcal{Z} \rightarrow B(\mathcal{V})$ satisfying $(*)$, one can build a structure of Lie algebra on $\mathcal{V}+\mathcal{Z}$. If $J \equiv 3(\bmod 4)$, there are several non isomorphic groups $\mathbb{H}_{K, J}$ for every admissible $K>K_{J}$ and only one $\mathbb{H}_{K_{J}, J}[\mathbf{B T V}, 3.1 .2]$.

The value of $K_{J}$ is computed as follows. By $[\mathbf{K}]$, the couple $(K, J)$ satisfies

$J<\rho(K)=8 \alpha+2^{\beta}$ where $K=m 2^{4 \alpha+\beta}, \quad m$ odd $, \quad 0 \leq \beta \leq 3, \quad \alpha, \beta \in \mathbb{N}$. 
In particular, $K$ must be even. Let $\rho_{J}$ be the smallest integer such that

$$
\rho_{J}=8 \alpha_{J}+2^{\beta_{J}}>J, \quad 0 \leq \beta_{J} \leq 3, \quad \alpha_{J}, \beta_{J} \in \mathbb{N}
$$

and let

$$
K_{J}=2^{4 \alpha_{J}+\beta_{J}}, \text { hence } \rho\left(K_{J}\right)=\rho_{J} .
$$

This ensures the existence of $\mathbb{H}_{K_{J}, J}$ and $K_{J}$ is the minimal possible dimension of $\mathcal{V}$, i.e. the dimension of each $\mathcal{V}_{l}$. In particular $J<K_{J}$.

For a given $\mathbb{H}_{K, J}$ we choose an orthonormal basis $X_{1}, \ldots, X_{K}$ of $\mathcal{V}$ as above. We denote by $g=(x, u)$ an element of $\mathbb{H}_{K, J}$, where $x=$ $\left(x_{k}\right)_{k=1}^{N K_{J}}$ are the coordinates corresponding to $X_{1}, \ldots, X_{K}, u=\left(u_{j}\right)_{j=1}^{J}$ are the coordinates corresponding to $U_{1}, \ldots, U_{J}$, and by $y_{l} \in \mathbb{R}^{K_{J}}$ the coordinates of $x$ corresponding to the basis of $\mathcal{V}_{l}$ or of $\mathcal{V}_{l}^{\prime}, 1 \leq l \leq N$, so that $x=\left(y_{1}, \ldots, y_{N}\right)$.

By definition [BTV, 3.1.5], $U_{j}=\frac{\partial}{\partial u_{j}}, 1 \leq j \leq J$, and

$$
X_{i}=\frac{\partial}{\partial x_{i}}+\frac{1}{2} \sum_{j=1}^{J}\left\langle\left[\sum_{k=1}^{K} x_{k} X_{k}, X_{i}\right], U_{j}\right\rangle \frac{\partial}{\partial u_{j}}, \quad 1 \leq i \leq K .
$$

In particular, if $X_{i}$ belongs to $\mathcal{V}_{l}$ or to $\mathcal{V}_{l}^{\prime}$, it only depends on $y_{l}$.

The heat kernel $p=p^{(K, J)}$ has properties analogous to those of $p^{(n)}$. First, $p^{(K, J)}$ is radial both with respect to $x$ and $u$ and depends only on $(K, J)$. This follows from the following formula established by J. Randall [R, Proof of Lemma 1.3.3]:

$$
\left(\mathcal{F}_{u} p^{(K, J)}\right)(x, \lambda)=\left(\mathcal{F}_{v} p^{\left(\frac{K}{2}\right)}\right)(x,|\lambda|), \quad \lambda \in \mathbb{R}^{J},
$$

where $\mathcal{F}_{u}$ denotes the Fourier transform on $\mathbb{R}^{J}$ with respect to $u, \mathcal{F}_{v}$ the Fourier transform on $\mathbb{R}$ with respect to $v \in \mathbb{R}$, and $p^{\left(\frac{K}{2}\right)}$ denotes the heat kernel on the Heisenberg goup $\mathbb{H}_{\frac{K}{2}}$. In particular, there is a unique $p^{\left(K_{J}, J\right)}$ for any given $J$.

We claim that

$$
p^{(K, J)}(x, u)=p^{\left(K_{J}, J\right)}\left(y_{1}, u\right) *_{u} \ldots *_{u} p^{\left(K_{J}, J\right)}\left(y_{N}, u\right)
$$

where convolution is on $\mathbb{R}^{J}$, with respect to $u$. This follows from Randall's formula and the fact that

$$
\left(\mathcal{F}_{v} p^{\left(\frac{K}{2}\right)}\right)(x,|\lambda|)=\Pi_{l=1}^{N}\left(\mathcal{F}_{v} p^{\left(\frac{K_{J}}{2}\right)}\right)\left(y_{l},|\lambda|\right) .
$$

It can also be proved directly. Indeed, let us denote by $q$ the convolution product on the right hand side. Since $p^{\left(K_{J}, J\right)}$ is a positive function with norm 1 in $L^{1}\left(\mathbb{R}^{K_{J}+J}\right), q$ is a positive function with norm 1 in $L^{1}\left(\mathbb{R}^{K+J}\right)$. As well known (see e.g. $[\mathbf{F S}]$ ), the heat kernel $p_{t}^{(K, J)}$ is the only positive function with norm 1 in $L^{1}\left(\mathbb{R}^{K+J}\right)$ satisfying $\frac{\partial f}{\partial t}=-\frac{1}{2} L f$; moreover, 
$p_{t}^{(K, J)}=t^{-\left(\frac{K}{2}+J\right)} p^{(K, J)} \circ \delta_{t^{-\frac{1}{2}}}$. Hence $p^{(K, J)}$ is the only positive function with norm 1 in $L^{1}\left(\mathbb{R}^{K+J}\right)$ satisfying

$$
(K \mathrm{Id}-L) p+\sum_{k=1}^{K} x_{k} X_{k} p=-2 \sum_{j=1}^{J} U_{j}\left(u_{j} p\right)
$$

(see e.g. [LP1, Lemmas 2, 3]). So we only have to verify that $q$ satisfies this equation. The left term can be splitted as $\sum_{l=1}^{N} D_{l}(p)$ where

$$
D_{l}=K_{J} \mathrm{Id}+\sum_{i=1}^{K_{J}}-X_{i+(l-1) K_{J}}^{2}+x_{i+(l-1) K_{J}} X_{i+(l-1) K_{J}} .
$$

By our choice of coordinates, $D_{l}$ only acts on the $l^{\text {th }}$ factor of $q$. For the right term, we note that

$$
\begin{aligned}
& u_{j} q\left(y_{1}, \ldots, y_{N}, u\right) \\
= & \sum_{l=1}^{N} p^{\left(K_{J}, J\right)}\left(y_{1}, u\right) *_{u} \ldots *_{u}\left(u_{j} p^{\left(K_{J}, J\right)}\right)\left(y_{l}, u\right) *_{u} p^{\left(K_{J}, J\right)}\left(y_{l+1}, u\right) *_{u} \ldots,
\end{aligned}
$$

which is easily verified by Fourier transform with respect to $u$. So it remains to verify that, for $1 \leq l \leq N$,

$$
\left(D_{l} p^{\left(K_{J}, J\right)}\right)\left(y_{l}, u\right)=-2 \sum_{j=1}^{J} U_{j}\left(u_{j} p^{\left(K_{J}, J\right.}\right)\left(y_{l}, u\right) .
$$

These are the heat equations corresponding to each Lie algebra $\mathcal{V}_{l}+\mathcal{Z}$ or $\mathcal{V}_{l}^{\prime}+\mathcal{Z}$. Hence they are all satisfied by $p^{\left(K_{J}, J\right.}$ as we saw above (this is obvious when there are only $\mathcal{V}_{l}$ 's).

For $g=(x, u) \in \mathbb{H}_{K, J}$ let $r=|x|, \rho=|u|$. As in a), we may rewrite for $1 \leq i \leq K$,

$$
X_{i} p^{(K, J)}=\frac{x_{i}}{r} \frac{\partial p^{(K, J)}}{\partial r}+\frac{1}{2}\left\langle\left[\sum_{k=1}^{K} x_{k} X_{k}, X_{i}\right], \sum_{j=1}^{J} u_{j} U_{j}\right\rangle \frac{1}{\rho} \frac{\partial p^{(K, J)}}{\partial \rho},
$$

and

(7) $\sqrt{2 \pi}\left(\sum_{i=1}^{K}\left|R_{i}(f)(\gamma)\right|^{2}\right)^{\frac{1}{2}} \leq\left(\sum_{i=1}^{K}\left|\int_{G} x_{i} F(\gamma, g) \frac{1}{r} \frac{\partial p^{(K, J)}}{\partial r}(g) d g\right|^{2}\right)^{\frac{1}{2}}$

$$
+\frac{1}{2}\left(\sum_{i=1}^{K}\left|\int_{G} F(\gamma, g)\left\langle\left[\sum_{k=1}^{K} x_{k} X_{k}, X_{i}\right], \sum_{j=1}^{J} u_{j} U_{j}\right\rangle \frac{1}{\rho} \frac{\partial p^{(K, J)}}{\partial \rho}(g) d g\right|^{2}\right)^{\frac{1}{2}} .
$$


The $L^{q}(d \gamma)$ norm of the first term in the righthand side of (7) is estimated as in a), replacing $p^{(1)}$ by $p^{\left(K_{J}, J\right)}$; it is less than

$$
\begin{aligned}
& h_{q}\left\|x_{1}\right\|_{\left.L^{q^{\prime}\left(\mathbb{R}^{K_{J}+J}, \mid \frac{1}{x_{1}}\right.} \frac{\partial p^{\left(K_{J}, J\right)}}{\partial x_{1}} \mid d x d u\right)}\left\|\frac{1}{x_{1}} \frac{\partial p^{\left(K_{J}, J\right)}}{\partial x_{1}}\right\|_{L^{1}\left(\mathbb{R}^{K_{j}+J}\right)}^{\frac{1}{q}}\|f\|_{L^{q}\left(\mathbb{H}_{K, J}\right)} \\
& =C_{1}(q, J)\|f\|_{L^{q}\left(\mathbb{H}_{K, J}\right)}
\end{aligned}
$$

and the constant is finite as in a) because $p^{\left(K_{J}, J\right)} \in \mathcal{S}\left(\mathbb{H}_{K_{J}, J}\right)$.

The second term is rewritten as

$$
\begin{array}{r}
\sup _{|a|=1} \int_{G} F(\gamma, g)\left\langle\left[\sum_{k=1}^{K} x_{k} X_{k}, \sum_{i=1}^{K} a_{i} X_{i}\right], \sum_{j=1}^{J} u_{j} U_{j}\right\rangle \frac{1}{\rho} \frac{\partial p^{(K, J)}}{\partial \rho}(g) d g \\
\leq A_{q^{\prime}}\|F(\gamma, .)\|_{L^{q}\left(\frac{1}{\rho}\left|\frac{\partial p^{(K, J)}}{\partial \rho}(g)\right| d g\right)}
\end{array}
$$

where

$$
A_{q^{\prime}}=\sup _{|a|=1}\left\|\sum_{j=1}^{J} u_{j}\left\langle\left[\sum_{k=1}^{K} x_{k} X_{k}, \sum_{i=1}^{K} a_{i} X_{i}\right], U_{j}\right\rangle\right\|_{L^{q^{\prime}}\left(\frac{1}{\rho}\left|\frac{\partial p(K, J)}{\partial \rho}(g)\right| d g\right)} .
$$

Hence, by Lemma 4 a), the $L^{q}(d \gamma)$ norm of the second term in the righthand side of (7) is estimated by

$$
\begin{aligned}
A_{q^{\prime}} h_{q}\left\|\frac{1}{u_{1}} \frac{\partial p^{(K, J)}}{\partial u_{1}}\right\|_{L^{1}\left(\mathbb{R}^{K+J}\right)}^{\frac{1}{q}}\|f\|_{L^{q}\left(\mathbb{H}_{K, J}\right)} & \\
& \leq A_{q^{\prime}} h_{q}\left\|\frac{1}{u_{1}} \frac{\partial p^{\left(K_{J}, J\right)}}{\partial u_{1}}\right\|_{L^{1}\left(\mathbb{R}^{K_{J}}+J\right)}^{\frac{1}{q}}\|f\|_{L^{q\left(\mathbb{H}_{K, J}\right)}} .
\end{aligned}
$$

We now estimate $A_{q^{\prime}}$. By Lemma 2 a) applied to $u_{1}, \ldots, u_{J}$ and fixed $x$,

$$
\begin{aligned}
\left\|\sum_{j=1}^{J} u_{j}\left\langle\left[\sum_{k=1}^{K} x_{k} X_{k}, \sum_{i=1}^{K} a_{i} X_{i}\right], U_{j}\right\rangle\right\|_{L^{q^{\prime}}\left(\frac{1}{\rho}\left|\frac{\partial p(K, J)}{\partial \rho}\right| d u\right)} \\
=B_{a}(x)\left\|u_{1}\right\|_{L^{q^{\prime}}\left(\frac{1}{\rho}\left|\frac{\partial p(K, J)}{\partial \rho}\right| d u\right)}
\end{aligned}
$$


where, since $\left|\sum_{i=1}^{K} a_{i} X_{i}\right|=1$,

$$
\begin{aligned}
B_{a}(x) & =\left|\left[\sum_{k=1}^{K} x_{k} X_{k}, \sum_{i=1}^{K} a_{i} X_{i}\right]\right|=\left|\operatorname{ad}_{\sum_{i=1}^{K} a_{i} X_{i}}\left(\sum_{k=1}^{K} x_{k} X_{k}\right)\right| \\
& =\left|P_{E_{a}}\left(\sum_{k=1}^{K} x_{k} X_{k}\right)\right|
\end{aligned}
$$

with $E_{a}=\left(\operatorname{ker} \operatorname{ad}_{\sum_{i=1}^{K} a_{i} X_{i}}\right)^{\perp}$. By rotation on the $x$ variables, we may suppose that $E_{a}$ is the span of $X_{1}, \ldots, X_{J}$. Since $\frac{\partial p^{(K, J)}}{\partial \rho}$ is radial with respect to $x$

$$
\begin{aligned}
A_{q^{\prime}}^{q^{\prime}} & =\sup _{|a|=1} \int_{\mathbb{R}^{K+J}}\left|B_{a}(x)\right|^{q^{\prime}}\left|u_{1}\right|^{q^{\prime}} \frac{1}{\rho}\left|\frac{\partial p^{(K, J)}}{\partial \rho}(x, u)\right| d x d u \\
& =\int_{\mathbb{R}^{K+J}}\left(\sum_{k=1}^{J}\left|x_{k}\right|^{2}\right)^{\frac{q^{\prime}}{2}}\left|u_{1}\right|^{q^{\prime}-1}\left|\frac{\partial p^{(K, J)}}{\partial u_{1}}(x, u)\right| d x d u \\
& \leq \int_{\mathbb{R}^{K_{J}+J}}\left(\sum_{k=1}^{J}\left|x_{k}\right|^{2}\right)^{\frac{q^{\prime}}{2}}\left|u_{1}\right|^{q^{\prime}-1}\left|\frac{\partial p^{\left(K_{J}, J\right)}}{\partial u_{1}}(x, u)\right| d x d u
\end{aligned}
$$

where the inequality is verified as in a), replacing $p^{(1)}$ by $p^{\left(K_{J}, J\right)}$, since $J<K_{J}$. Finally

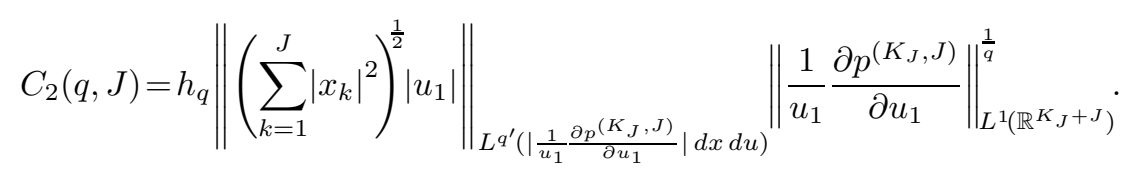

This constant is finite because $p^{\left(K_{J}, J\right)} \in \mathcal{S}\left(\mathbb{H}_{K_{J}, J}\right)$ and we get $\left\|\left(\sum_{i=1}^{K}\left|R_{i}(f)(\gamma)\right|^{2}\right)^{\frac{1}{2}}\right\|_{L^{q}\left(\mathbb{H}_{K, J}\right)} \leq \frac{1}{\sqrt{2 \pi}}\left(C_{1}(q, J)+\frac{1}{2} C_{2}(q, J)\right)\|f\|_{L^{q}\left(\mathbb{H}_{K, J}\right)}$.

This implies as in a) the lefthand side inequality. 
Remark. The above proof of a) does not seem to extend to the setting of non isotropic Heisenberg groups, because it uses in a crucial way the radiality of $p$; so does the proof in $[\mathbf{C M Z}]$. However, it is stated in [BDJ, p. 59], without explanation, that the proof in $[\mathbf{C M Z}]$ can be extended to the non isotropic case.

\section{Riesz transforms associated to commuting inner $*$-derivations}

\subsection{Introduction and notation.}

We now show how the classical result on Riesz transforms on $L^{q}\left(\mathbb{R}^{n}, d x\right)$ can be extended to Riesz transforms on Schatten spaces $\mathcal{S}^{q}(H)$, defined by inner (bounded or unbounded) commuting $*$-derivations acting on the $\mathrm{C}^{*}$ algebra $K(H)$ of compact operators on the Hilbert space $H$.

We denote by $\gamma_{n}$ the standard gaussian density on $\mathbb{R}^{n}$ and by $\Pi$ the orthogonal projection onto the linear span of the coordinates $y_{j}$ in $L^{2}\left(\gamma_{n}\right)$. As well known, $\Pi$ extends as a bounded operator: $L^{q}\left(\gamma_{n}\right) \rightarrow$ $L^{q}\left(\gamma_{n}\right), 1 \leq q<\infty$, which we still denote by $\Pi$.

The Fourier transform on $\mathbb{R}$ is defined by $\widehat{f}(u)=\int_{\mathbb{R}} e^{-i y u} f(y) d y$.

We recall that $\mathcal{S}^{q}(H)$ is the space of compact operators $X$ on $H$ such that $\operatorname{tr}\left(X^{*} X\right)^{\frac{q}{2}}<\infty, 1 \leq q<\infty . B(H)$ is the dual space of $\mathcal{S}^{1}(H)$ which is itself the dual space of $K(H) \cdot \mathcal{S}^{2}(H)$ is the Hilbert space of Hilbert Schmidt operators on $H$. From now on, we assume $H$ is separable.

We denote by $|X|_{s}^{2}=X^{*} X+X X^{*}$ the symmetrized modulus of $X \in$ $K(H)$.

Let $h$ be a $*$-automorphism of $K(H)$, hence $h^{* *}$ is a *-automorphism of $B(H)$. By [Pe, Theorem 8.9.2] there exists a unitary operator $U$ on $H$ such that $h(X)=U X U^{*}, X \in K(H)$. Let $\left(h_{y}\right)_{y \in \mathbb{R}}$ be a strongly continuous one parameter group of $*$-automorphisms of $K(H)$; then there exists a one parameter group of unitaries $\left(U_{y}\right)_{y \in \mathbb{R}}$ such that $h_{y}(X)=U_{y} X U_{y}^{*}$, $X \in K(H)$ (see e.g. [V $\mathbf{V}$ Theorem 11.1] for an actually stronger result, or [Par, pp. 86-87]); by Stone's theorem $U_{y}=e^{y A}$, where $i A$ is selfadjoint on $H$. Hence the generator $D$ of $\left(h_{y}\right)_{y \in \mathbb{R}}$ is an inner $*$-derivation, in general unbounded, defined by $D(X)=[A, X]$. In particular $\left(h_{y}\right)_{y \in \mathbb{R}}$ is a group of isometries of $\mathcal{S}^{2}(H)$ (and a group of isometries of every $\left.\mathcal{S}^{q}(H), 1 \leq q<\infty\right)$. By [RS, Theorem VIII.9], $\left(h_{y}\right)_{y \in \mathbb{R}}$ is strongly continuous on $\mathcal{S}^{2}(H)$. Its generator is naturally induced by $D$, and $i D$ is self-adjoint on $\mathcal{S}^{2}(H)$ by Stone's theorem.

Let us now recall some facts about joint functional calculus for "commuting" *-inner derivations. We consider $n$ strongly continuous one 
parameter groups of $*$-automorphisms of $K(H)$, with respective generators $D_{j}, 1 \leq j \leq n$, which we denote respectively by $\left(e^{y_{j} D_{j}}\right)_{y_{j} \in \mathbb{R}}$. We assume that

(i) their restrictions commute on $\mathcal{S}^{2}(H)$

(ii) the only $X \in \mathcal{S}^{2}(H)$ which is invariant under every $e^{y_{j} D_{j}}$ is $X=0$.

In particular, by (i),

$$
y \longrightarrow U(y)=e^{\sum_{j=1}^{n} y_{j} D_{j}}
$$

is a strongly continuous map of $\mathbb{R}^{n}$ into the unitary operators on $\mathcal{S}^{2}(H)$, satisfying $U(y+z)=U(y) U(z), y, z \in \mathbb{R}^{n}$ and $U(0)=I$. Then [RS, Theorem VIII.12], there is a projection valued measure $E$ on $\mathbb{R}^{n}$ such that

(8) $\left\langle e^{\sum_{j=1}^{n} y_{j} D_{j}}(X), Y\right\rangle=\int_{\mathbb{R}^{n}} e^{i\langle y, \lambda\rangle} d\left\langle E_{\lambda}(X), Y\right\rangle$,

$$
X, Y \in \mathcal{S}^{2}(H), \quad y \in \mathbb{R}^{n} .
$$

By bounded functional calculus and Fubini theorem, for every $F \in$ $\mathcal{S}\left(\mathbb{R}^{n}\right)$, as bounded operators on $\mathcal{S}^{2}(H)$ [RS, p. 272],

$$
\widehat{F}\left(i D_{1}, \ldots, i D_{n}\right)=\int_{\mathbb{R}^{n}} e^{\sum_{k=1}^{n} y_{k} D_{k}} F(y) d y .
$$

Moreover, let $h: \mathbb{R}^{n} \rightarrow \mathbb{R}$ be a Borel measurable function and let $X \in$ $\mathcal{S}^{2}(H)$ be such that $\int_{\mathbb{R}^{n}}|h(\lambda)|^{2} d\left\langle E_{\lambda}(X), X\right\rangle<\infty$; then the formula

$$
\left\langle h\left(i D_{1}, \ldots, i D_{n}\right)(X), Y\right\rangle=\int_{\mathbb{R}^{n}} h(\lambda) d\left\langle E_{\lambda}(X), Y\right\rangle, \quad Y \in \mathcal{S}^{2}(H)
$$

defines an operator $h\left(i D_{1}, \ldots, i D_{n}\right)$ which is densely defined and selfadjoint on $\mathcal{S}^{2}(H)$. This holds in particular for

$$
L=-\sum_{k=1}^{n} D_{k}^{2}
$$

By assumption (ii), the projection $E_{\{0\}}$ is null. Indeed, let $Z \in \mathcal{S}^{2}(H)$ lying in its range. Since the measure $E-\delta_{0} \otimes E_{\{0\}}$ is valued in the set of orthogonal projections $P$ on $\mathcal{S}^{2}(H)$ such that $P E_{\{0\}}=0$, hence $P(Z)=$ 0 , the scalar measure $1_{\left\{\mathbb{R}^{n} \backslash\{0\}\right\}}(\lambda) d\left\langle E_{\lambda}(Z), Y\right\rangle$ is zero for every $Y \in$ $\mathcal{S}^{2}(H)$; then, by (8),

$$
\left\langle Y, e^{\sum_{j=1}^{n} y_{j} D_{j}}(Z)\right\rangle=\left\langle e^{i\langle y, \lambda\rangle}, \delta_{0}\right\rangle\left\langle Y, E_{\{0\}} Z\right\rangle=\langle Y, Z\rangle,
$$

which, by (ii), implies $Z=0$. 
The Riesz transforms are defined by

$$
R_{j}=D_{j} L^{-\frac{1}{2}}, \quad 1 \leq j \leq n,
$$

so they are contractions on their domain in $\mathcal{S}^{2}(H)$. Since $E_{\{0\}}=0, R_{j}$ is actually defined on $\mathcal{S}^{2}(H)$ by

$$
\left\langle R_{j}(X), Y\right\rangle=\int_{\mathbb{R}^{n} \backslash\{0\}} \frac{i \lambda_{j}}{|\lambda|} d\left\langle E_{\lambda}(X), Y\right\rangle .
$$

The main result of this part is the following theorem:

Theorem 5. Let $H$ be a separable Hilbert space and let $\left(e^{y_{j} D_{j}}\right)_{y_{j} \in \mathbb{R}, 1 \leq}$ $j \leq n$ be strongly continuous one parameter groups of $*$-automorphisms of $K(H)$ satisfying the above conditions (i), (ii). Let $1<q<\infty$.

a) Then, for every $X \in K(H)$ and $t \in \mathbb{R}$,

$$
e^{-\frac{1}{2} t^{2} L}(X)=\int_{\mathbb{R}^{n}} e^{t \sum_{j=1}^{n} y_{j} D_{j}}(X) \gamma_{n}(y) d y
$$

$e^{-\frac{1}{2} t^{2} L}$ is a completely positive contraction: $K(H) \rightarrow K(H)$ and $a$ contraction of every $\mathcal{S}^{q}(H)$.

b) The operator $\mathcal{R}=\sum_{j=1}^{n} y_{j} R_{j}$ is a (completely) bounded operator: $\mathcal{S}^{q}(H) \rightarrow L^{q}\left(\gamma_{n}(y) d y, \mathcal{S}^{q}\right)$ which satisfies

$$
\sqrt{2 \pi} \mathcal{R}(X)=\left(\Pi \otimes I_{\mathcal{S} q}\right)\left(p v \int_{-\infty}^{\infty} e^{t \sum_{j=1}^{n} y_{j} D_{A_{j}}}(X) \frac{d t}{t}\right) .
$$

c) For $X \in \mathcal{S}^{q},\|X\|_{\mathcal{S}^{q}}$ is respectively equivalent, with constants which depend only on $q$, to

(i) $\left\|\left(\sum_{j=1}^{n}\left|R_{j}(X)\right|_{s}^{2}\right)^{\frac{1}{2}}\right\|_{\mathcal{S}^{q}}, \quad 2 \leq q<\infty$,

and to

(ii) $\inf \left\{\left\|\left(\sum_{j=1}^{n} B_{j} B_{j}^{*}\right)^{\frac{1}{2}}\right\|_{\mathcal{S}^{q}}+\left\|\left(\sum_{j=1}^{n} C_{j}^{*} C_{j}\right)^{\frac{1}{2}}\right\|_{\mathcal{S}^{q}}\right\}, \quad 1<q<2$, where the infimum is taken over all decompositions $R_{j}(X)=B_{j}+$ $C_{j}$ in $\mathcal{S}^{q}(H)$.

Note that, on $H$, the operators $R_{j}(X), 1 \leq j \leq n$, do not commute in general, and $R_{j}(X)^{*}=R_{j}\left(X^{*}\right)$ does not commute in general with $R_{j}(X)$. 
Proof of Theorem 5: The strategy is similar to Pisier's in the first part of $[\mathbf{P 1}]$ and to the one we used in the first part of this paper. On one hand, things are much easier than for Heisenberg groups because we deal with a one parameter group; on the other hand, some difficulties arise from the setting of non commutative $L^{q}$ 's.

a) Formula (9) defines bounded operators on $K(H)$ and $\mathcal{S}^{q}(H), 1 \leq$ $q<\infty$, with norm less than $\|F\|_{L^{1}(d y)}$, because $e^{\sum_{k=1}^{n} y_{k} D_{k}}$ is an isometry of $K(H)$ and $\mathcal{S}^{q}(H)$ for every $y$.

This formula gives a Stinespring factorization (see e.g. $[\mathbf{P a}]$ ) of $\widehat{F}\left(i D_{1}, \ldots, i D_{n}\right)$ acting on $K(H)$ because

$$
X \longrightarrow e^{\sum_{j=1}^{n} y_{j} D_{j}}(X)
$$

is a *-homomorphism: $K(H) \rightarrow L^{\infty}\left(d \gamma_{n}, B(H)\right) \subset B\left(L^{2}\left(d \gamma_{n}, H\right)\right)$.

Applying this to $F(y)=\gamma_{n}(y)$ and $t D_{1}, \ldots, t D_{n}$ proves assertion a).

b) $\alpha$ ) By (10) and Fubini theorem, for $X \in \mathcal{S}^{2}(H)$,

$$
\begin{aligned}
\sqrt{\frac{\pi}{2}}\left\langle R_{j}(X), X\right\rangle & =\sqrt{\frac{\pi}{2}} \int_{\mathbb{R}^{n}} \frac{i \lambda_{j}}{|\lambda|} d\left\langle E_{\lambda}(X), X\right\rangle \\
& =\int_{\mathbb{R}^{n}} i \lambda_{j}\left(\int_{0}^{\infty} e^{-\frac{1}{2} t^{2}|\lambda|^{2}} d t\right) d\left\langle E_{\lambda}(X), X\right\rangle \\
& =\int_{0}^{\infty}\left(\int_{\mathbb{R}^{n}} i \lambda_{j} e^{-\frac{1}{2} t^{2}|\lambda|^{2}} d\left\langle E_{\lambda}(X), X\right\rangle\right) d t \\
& =\int_{0}^{\infty}\left\langle D_{j} e^{-\frac{1}{2} t^{2} L}(X), X\right\rangle d t
\end{aligned}
$$

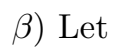

$$
F(y)=y_{j} \gamma_{n}(y), \text { hence } \widehat{F}(u)=-i u_{j} e^{-\frac{1}{2}|u|^{2}} .
$$

By (9) applied to $F$ and $t D_{1}, \ldots, t D_{n}$, by symmetry with respect to $t$, for $X \in \mathcal{S}^{q}(H)$,

$$
\begin{aligned}
t D_{j} e^{-\frac{1}{2} t^{2} L}(X) & =\int_{\mathbb{R}^{n}} e^{t \sum_{k=1}^{n} y_{k} D_{k}}(X) y_{j} \gamma_{n}(y) d y \\
& \left.=\frac{1}{2} \int_{\mathbb{R}^{n}}\left(e^{t \sum_{k=1}^{n} y_{k} D_{k}}(X)\right)-e^{-t \sum_{k=1}^{n} y_{k} D_{k}}(X)\right) y_{j} \gamma_{n}(y) d y
\end{aligned}
$$


Hence, by $\alpha$ ), for $X \in \mathcal{S}^{2}(H)$,

$$
\begin{aligned}
\sqrt{2 \pi} R_{j}(X)=\int_{0}^{\infty} & {\left[\int _ { \mathbb { R } ^ { n } } \left(e^{t \sum_{k=1}^{n} y_{k} D_{k}}(X)\right.\right.} \\
& \left.\left.-e^{-t \sum_{k=1}^{n} y_{k} D_{k}}(X)\right) y_{j} \gamma_{n}(y) d y\right] \frac{d t}{t} \\
=\int_{-\infty}^{\infty} & {\left[\int_{\mathbb{R}^{n}} e^{t \sum_{k=1}^{n} y_{k} D_{k}}(X) y_{j} \gamma_{n}(y) d y\right] \frac{d t}{t} . }
\end{aligned}
$$

$\gamma$ ) We claim that, for every $X \in \mathcal{S}^{q}(H), y \in \mathbb{R}^{n}, 1<q<\infty$,

$$
F(y, X)=\operatorname{pv} \int_{-\infty}^{\infty} e^{t \sum_{k=1}^{n} y_{k} D_{k}}(X) \frac{d t}{t}
$$

is well defined as the norm limit in $\mathcal{S}^{q}(H)$ of

$$
F(y, X, \varepsilon)=\int_{\varepsilon<|t|<\varepsilon^{-1}} e^{t \sum_{k=1}^{n} y_{k} D_{k}}(X) \frac{d t}{t}
$$

and satisfies

$$
\|F(y, X)\|_{\mathcal{S}^{q}} \leq h_{q}\|X\|_{\mathcal{S}^{q}} .
$$

Indeed, $\mathcal{S}^{q}(H)$ is UMD for $1<q<\infty$ [BGM, Theorem 6.1] and, for fixed $y,\left(e^{t \sum_{k=1}^{n} y_{k} D_{k}}\right)_{t \in \mathbb{R}}$ is a strongly continuous one parameter group of isometries of $\mathcal{S}^{q}(H)$. Hence [BGM, Theorems 5.12 and 5.16] prove the claim.

$\delta$ ) It follows that, for the $\mathcal{S}^{2}(H)$ norm, by Fubini theorem and (11),

$$
\begin{aligned}
\int_{\mathbb{R}^{n}} F(y, X) y_{j} \gamma_{n}(y) d y & =\lim _{\varepsilon} \int_{\mathbb{R}^{n}} F(y, X, \varepsilon) y_{j} \gamma_{n}(y) d y \\
& =\lim _{\varepsilon} \int_{\varepsilon<|t|<\varepsilon^{-1}}\left(\int_{\mathbb{R}^{n}} e^{t \sum_{k=1}^{n} y_{k} D_{k}}(X) y_{j} \gamma_{n}(y) d y\right) \frac{d t}{t} \\
& =\sqrt{2 \pi} R_{j}(X) .
\end{aligned}
$$

Hence, for $X \in \mathcal{S}^{q}(H) \cap \mathcal{S}^{2}(H)$,

$$
\sqrt{2 \pi} \mathcal{R}(X)=\sum_{j=1}^{n} y_{j} \int_{\mathbb{R}^{n}} F(y, X) y_{j} \gamma_{n}(y) d y=\left(\Pi \otimes I_{\mathcal{S}^{q}}\right)(F(y, X)),
$$

and, by (12),

$$
\|F(y, X)\|_{L^{q}\left(\gamma_{n} d y, \mathcal{S}^{q}\right)} \leq h_{q}\|X\|_{\mathcal{S}^{q}} .
$$


$\varepsilon)$ By $\left[\mathbf{P 2}\right.$, Remark 8.4.6], $\Pi \otimes I_{\mathcal{S}^{q}}$ is bounded on $L^{q}\left(\gamma_{n} d y, \mathcal{S}^{q}\right)$, $1<q<\infty$, and its norm depends only on $q$. By $\delta$ ) this proves the boundedness of $\mathcal{R}$ on $\mathcal{S}^{q}(H)$ and ends the proof of b).

Actually, $\Pi$ is completely bounded, hence so is $\Pi \otimes I_{\mathcal{S}^{q}}$, and the same argument as above, applied to $\left(e^{t \sum_{j=1}^{n} y_{j} D_{A_{j}}} \otimes I_{\mathcal{S}^{q}}\right)_{t \in \mathbb{R}}$ shows that, for fixed $y, \mathrm{pv} \int_{-\infty}^{\infty} e^{t \sum_{j=1}^{n} y_{j} D_{A_{j}}} \frac{d t}{t}$ is completely bounded: $\mathcal{S}^{q}(H) \rightarrow$ $\mathcal{S}^{q}(H)$.

c) By [P2, Theorem 8.4.1], the norms in the statement are equivalent, with constants which depend only on $q$, to

$$
\left\|\sum_{j=1}^{n} y_{j} R_{j}(X)\right\|_{L^{q}\left(d \gamma_{n}, \mathcal{S}^{q}\right)}=\|\mathcal{R}(X)\|_{L^{q}\left(d \gamma_{n}, \mathcal{S}^{q}\right)} .
$$

Hence, we have to show that $\|X\|_{\mathcal{S}^{q}}$ is equivalent to $\|\mathcal{R}(X)\|_{L^{q}\left(d \gamma_{n}, \mathcal{S}^{q}\right)}$. One inequality has been proved in $\mathrm{b})$. For the other one, we notice that $\mathcal{R}^{*} \mathcal{R}=\operatorname{Id}$ on $\mathcal{S}^{2}(H)$ because

$$
\begin{aligned}
\langle\mathcal{R}(X), \mathcal{R}(Y)\rangle & =\int_{\mathbb{R}^{n}}\left\langle\sum_{j=1}^{n} y_{j} R_{j}(X), \sum_{k=1}^{n} y_{k} R_{k}(Y)\right\rangle \gamma_{n}(y) d y \\
& =\sum_{j=1}^{n}\left\langle R_{j}(X), R_{j}(Y)\right\rangle=\sum_{j=1}^{n}\left\langle R_{j}^{*} R_{j}(X), Y\right\rangle=\langle X, Y\rangle .
\end{aligned}
$$

Hence, for $\frac{1}{q}+\frac{1}{q^{\prime}}=1$,

$\|X\|_{\mathcal{S}^{q}} \leq\left\|\mathcal{R}^{*}\right\|_{q \rightarrow q}\|\mathcal{R}(X)\|_{L^{q}\left(\gamma_{n} d y, \mathcal{S}^{q}\right)}=\|\mathcal{R}\|_{q^{\prime} \rightarrow q^{\prime}}\|\mathcal{R}(X)\|_{L^{q}\left(\gamma_{n} d y, \mathcal{S}^{q}\right)}$.

Example 6. The CCR heat flow on $K\left(L^{2}\left(\mathbb{R}^{n}\right)\right)$.

The assumptions of Theorem 5 are satisfied by the following example, taken from $[\mathbf{A}]$, where assertion a) is proved in this special case.

The operator $P=-i \frac{d}{d x}$ and the operator $Q$ of multiplication by $x$ are well defined: $\mathcal{S}(\mathbb{R}) \rightarrow \mathcal{S}(\mathbb{R})$ and satisfy the Canonical Commutation Relation

$$
[Q, P]=i I \text {. }
$$

$P, Q$ are formally selfadjoint unbounded operators on $L^{2}(\mathbb{R})$. They generate two one parameter unitary groups of operators on $L^{2}(\mathbb{R})$, respectively $e^{i s P}$ (translation by $-s$ ) and $e^{i t Q}$ (multiplication by $e^{i t x}$ ), satisfying

$$
e^{i s P} e^{i t Q}=e^{i s t} e^{i t Q} e^{i s P}, \quad s, t \in \mathbb{R} .
$$


We may define the inner $*$-derivations

$$
\begin{aligned}
& D_{P}(X)=i[P, X], \\
& D_{Q}(X)=i[Q, X]
\end{aligned}
$$

for operators $X$ on $L^{2}(\mathbb{R})$ which are defined by kernels $k(x, y) \in \mathcal{S}\left(\mathbb{R}^{2}\right)$. Note that the kernels of $D_{P}(X), D_{Q}(X)$ are respectively $\left(\frac{\partial}{\partial x}+\frac{\partial}{\partial y}\right) k$ and $(x-y) k . \quad D_{P}, D_{Q}$ are generators of groups of $*$-automorphisms of $K\left(L^{2}(\mathbb{R})\right)$, namely

$$
\begin{gathered}
e^{s D_{P}}(X)=e^{i s P} X e^{-i s P} \\
e^{t D_{Q}}(X)=e^{i t Q} X e^{-i t Q}
\end{gathered}
$$

and, owing to (13), $e^{s D_{P}}$ and $e^{t D_{Q}}$ commute on $K\left(L^{2}(\mathbb{R})\right)$. Obviously, only $X=0$ is stable under these groups.

On $\mathcal{S}\left(\mathbb{R}^{n}\right)$ we consider in the same way $P_{j}=-i \frac{\partial}{\partial x_{j}}, Q_{j}$ the operator of multiplication by $x_{j}$. The $*$-automorphisms $e^{s_{j} D_{P_{j}}}$ and $e^{t_{k} D_{Q_{k}}}, 1 \leq$ $j, k \leq n$ all commute on $K\left(L^{2}\left(\mathbb{R}^{n}\right)\right)$. Denoting

$$
L=-\sum_{j=1}^{n} D_{P_{j}}^{2}+D_{Q_{j}}^{2},
$$

the semigroup $e^{-t L}, t \geq 0$, acting on $K\left(L^{2}\left(\mathbb{R}^{n}\right)\right)$, is called the CCR heat flow. The $2 n$ Riesz transforms $D_{P_{j}} L^{-\frac{1}{2}}, D_{Q_{k}} L^{-\frac{1}{2}}, 1 \leq j, k \leq n$ satisfy the conclusion of Theorem 5 .

\section{References}

[A] W. Arveson, The heat flow of the CCR algebra, Bull. London Math. Soc. 34(1) (2002), 73-83.

[ABS] M. F. АтiYah, R. Bott and A. Shapiro, Clifford modules, Topology 3, suppl. 1 (1964), 3-38.

[BDJ] C. Berenstein, Der-Chen Chang And Jingzhi Tie, " $L a-$ guerre calculus and its applications on the Heisenberg group", AMS/IP Studies in Advanced Mathematics 22, American Mathematical Society, Providence, RI; International Press, Somerville, MA, 2001.

[BGM] E. Berkson, T. A. Gillespie And P. S. Muhly, Abstract spectral decompositions guaranteed by the Hilbert transform, Proc. London Math. Soc. (3) 53(3) (1986), 489-517. 
[BTV] J. Berndt, F. Tricerri and L. Vanhecke, "Generalized Heisenberg groups and Damek-Ricci harmonic spaces", Lecture Notes in Mathematics 1598, Springer-Verlag, Berlin, 1995.

[C] M. Christ, Hilbert transforms along curves. I. Nilpotent groups, Ann. of Math. (2) 122(3) (1985), 575-596.

[CMZ] T. Coulhon, D. Müller and J. Zienkiewicz, About Riesz transforms on the Heisenberg groups, Math. Ann. 305(2) (1996), 369-379.

[CDKR] M. Cowling, A. H. Dooley, A. Korányi and F. Ricci, $H$-type groups and Iwasawa decompositions, Adv. Math. 87(1) (1991), 1-41.

[CY] J. CYGan, Heat kernels for class 2 nilpotent groups, Studia Math. 64(3) (1979), 227-238.

[FS] G. B. Folland and E. M. Stein, "Hardy spaces on homogeneous groups", Mathematical Notes 28, Princeton University Press, Princeton, N.J.; University of Tokyo Press, Tokyo, 1982.

[G] B. Gaveau, Principe de moindre action, propagation de la chaleur et estimées sous elliptiques sur certains groupes nilpotents, Acta Math. 139(1-2) (1977), 95-153.

$[\mathrm{H}]$ A. Hulanicki, The distribution of energy in the Brownian motion in the Gaussian field and analytic-hypoellipticity of certain subelliptic operators on the Heisenberg grou, Studia Math. 56(2) (1976), 165-173.

[Hu] D. Husemoller, "Fibre bundles", Third edition, Graduate Texts in Mathematics 20, Springer-Verlag, New York, 1994.

[K] A. Kaplan, Fundamental solutions for a class of hypoelliptic PDE generated by composition of quadratic forms, Trans. Amer. Math. Soc. 258(1) (1980), 147-153.

[LP1] F. Lust-Piquard, A simple minded computation of heat kernels on Heisenberg groups, Colloq. Math. 97(2) (2003), 233-249.

[LP2] F. Lust-PiQuARD, Dimension free estimates for discrete Riesz transforms on products of abelian groups, Adv. Math. (to appear).

[LP3] F. Lust-PiQuard, Riesz transforms associated with the number operator on the Walsh system and the fermions, J. Funct. Anal. 155(1) (1998), 263-285.

[Par] K. R. Parthasarathy, "An introduction to quantum stochastic calculus", Monographs in Mathematics 85, Birkhäuser Verlag, Basel, 1992. 
[Pa] V. I. PAulsen, "Completely bounded maps and dilations", Pitman Research Notes in Mathematics Series 146, Longman Scientific \& Technical, Harlow; John Wiley \& Sons, Inc., New York, 1986.

[Pe] G. K. Pedersen, "C*-algebras and their automorphism groups", London Mathematical Society Monographs 14, Academic Press, Inc. [Harcourt Brace Jovanovich, Publishers], London-New York, 1979.

[P1] G. PISIER, Riesz transforms: a simpler analytic proof of P.-A. Meyer's inequality, in: "Séminaire de Probabilités, XXII", Lecture Notes in Math. 1321, Springer, Berlin, 1988, pp. $485-501$.

[P2] G. PIsIER, Non-commutative vector valued $L_{p}$-spaces and completely p-summing maps, Astérisque 247 (1998), 131 pp.

[R] J. RANDALL, The heat kernel for generalized Heisenberg groups, J. Geom. Anal. 6(2) (1996), 287-316.

[RS] M. REED AND B. Simon, "Methods of modern mathematical physics. I. Functional analysis", Second edition, Academic Press, Inc. [Harcourt Brace Jovanovich, Publishers], New York, 1980.

[S] E. M. STEIn, Some results in harmonic analysis in $\mathbb{R}^{n}$, for $n \rightarrow \infty$, Bull. Amer. Math. Soc. (N.S.) 9(1) (1983), 71-73.

[V] V. S. VARADARAJAn, "Geometry of quantum theory", Vol. II, The University Series in Higher Mathematics, Van Nostrand Reinhold Co., New York-Toronto, Ont.-London, 1970.

Département de Mathématiques

Université de Cergy

2 Av. A. Chauvin

95302 Cergy

France

E-mail address: Francoise.Piquard@math.u-cergy.fr 
